This item was submitted to Loughborough's Research Repository by the author.

Items in Figshare are protected by copyright, with all rights reserved, unless otherwise indicated.

\title{
Modeling the electromigration failure time distribution in short copper interconnects
}

PLEASE CITE THE PUBLISHED VERSION

PUBLISHER

(C) American Institute of Physics

\section{VERSION}

VoR (Version of Record)

LICENCE

CC BY-NC-ND 4.0

\section{REPOSITORY RECORD}

Dwyer, Vincent M.. 2019. "Modeling the Electromigration Failure Time Distribution in Short Copper Interconnects". figshare. https://hdl.handle.net/2134/4986. 
This item was submitted to Loughborough's Institutional Repository (https://dspace.lboro.ac.uk/) by the author and is made available under the following Creative Commons Licence conditions.

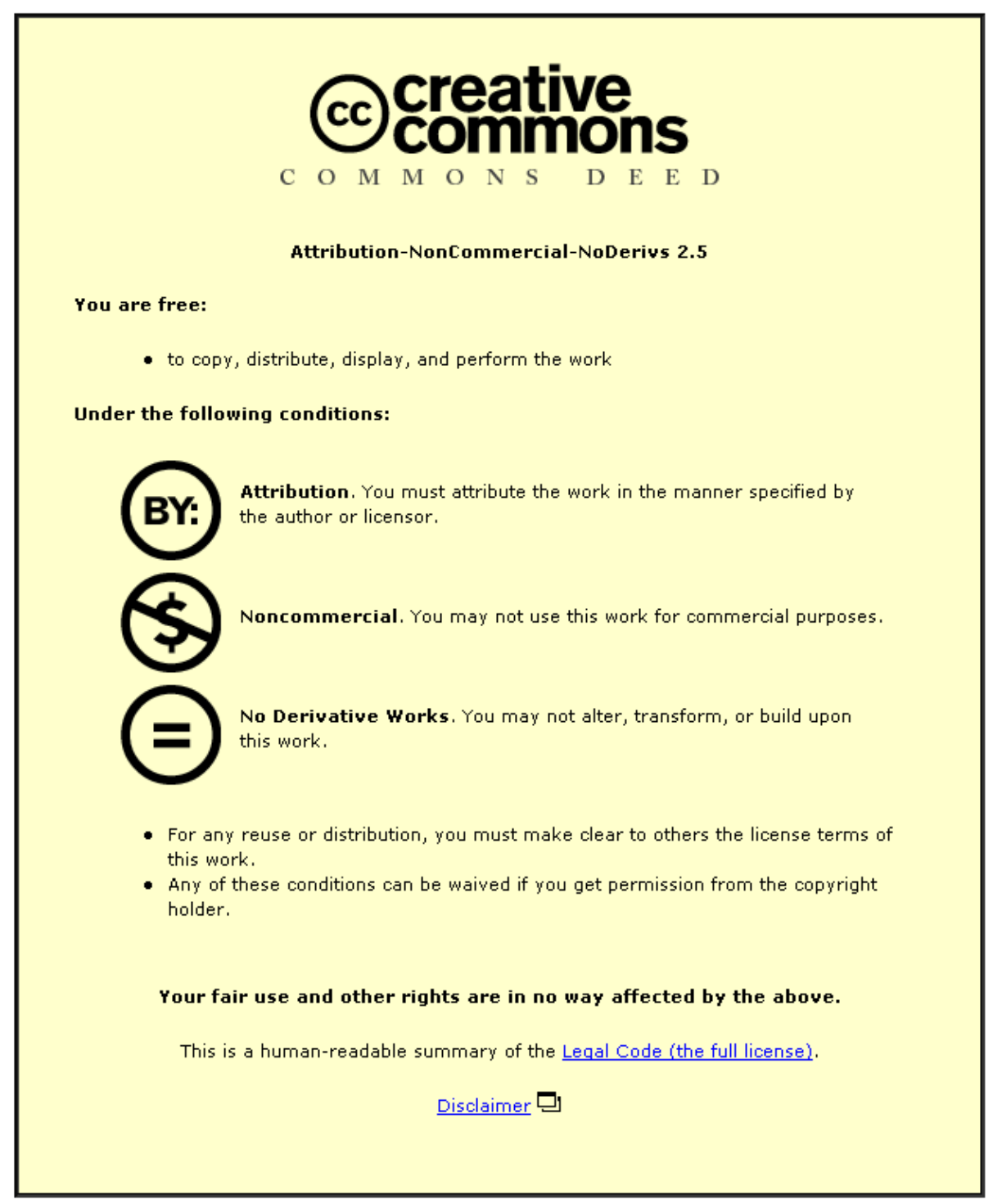

For the full text of this licence, please go to: http://creativecommons.org/licenses/by-nc-nd/2.5/ 


\title{
Modeling the electromigration failure time distribution in short copper interconnects
}

\author{
V. M. Dwyer ${ }^{\mathrm{a})}$ \\ Department of Electronic and Electrical Engineering, Loughborough University, Loughborough LE11 3TU, \\ United Kingdom
}

(Received 26 March 2008; accepted 20 June 2008; published online 4 September 2008)

The electromigration (EM) lifetime in short copper interconnects is modeled using a previously developed means of generating realistic interconnect microstructures combined with the one-dimensional stress evolution equation of Korhonen et al. [J. Appl. Phys. 73, 3790 (1993)]. This initial analysis describes the void nucleation and subsequent growth in lines blocked at one end and terminated with a pad at the other. For short copper interconnects, the failure time is largely spent on void growth, and, for sufficiently short lines $(\$ 50 \mathrm{~mm})$, the growth is largely steady state. This allows for the development of a simple expression for the variation of the failure time with microstructure. Assuming that the diffusion activation energies are normally distributed, the permanence property of summed lognormals leads to a roughly lognormal distribution for EM failure times. Importantly for EM design rules, linear extrapolation on lognormal plot is found to slightly underestimate interconnect reliability. (C) 2008 American Institute of Physics.

[DOI: $10.1063 / 1.2970171]$

\section{INTRODUCTION}

Electromigration (EM) is still one of the most serious reliability concerns for integrated circuit (IC) metallization made worse with each new technology node. Large current densities transfer momentum from conduction electrons to metal atoms causing migration toward the interconnect anode. In general, the cathode is unable to replace the migrating atoms, usually due to the presence of a refractory metal barrier between two metal interconnect layers, making the cathode a likely site for void nucleation and growth and eventual line failure. EM damage can also occur within lines at points of flux divergence; the triple points at the cathode end of grain boundary clusters in near-bamboo aluminum interconnect provide an obvious example. Recent results in narrow copper lines demonstrate that the grain orientations on the cathode and anode sides of in-line voids show distinctive trends. ${ }^{1}$ On the cathode side, $\left(\begin{array}{lll}1 & 1 & 1\end{array}\right)$ grains or one of its

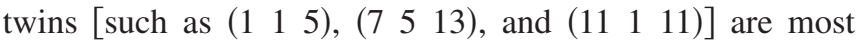
commonly found, while on the anode side the grains tend to be either an unrelated orientation or a more distant $\left(\begin{array}{lll}1 & 1 & 1\end{array}\right)$ twin than that found on the cathode side. This suggests both that $\left(\begin{array}{lll}1 & 1 & 1\end{array}\right)$ grains provide the slowest diffusion path and that such variations in the diffusivity on the scale of the individual grain size play an important role in the explanation and understanding of EM voiding and hence failure. Nucleation may also occur at those defect sites capable of lowering the barrier to void nucleation. In copper such voids can be detached and drift to the cathode where they can grow to failure. Pre-existing voids formed during the fabrication process are also able to grow and similarly detach. Somewhat remarkably and irrespective of the metal used, the line dimensions, the integration scheme, the EM failure site, or the

\footnotetext{
${ }^{a)}$ Tel.: +44(0)1509 227027. FAX: +44(0)1509 227014. Electronic mail: v.m.dwyer@lboro.ac.uk.
}

degree of process control, experimentally obtained accelerated lifetime data provide a very reasonable fit on a lognormal plot (e.g., Refs. 2-5). Such that is its regard that even data which give a relatively poor fit to lognormality are generally assumed to be due to multimodality within the failures rather than to different failure statistics (e.g., Ref. 6).

It still remains something of an open question whether or not failure statistics are the same at test and use conditions. However, if lognormality of the failure time is appropriate and if the variations with the acceleration parameters (temperature and current density) of the median time to failure $t_{50}$ and the deviation in time to failure (DTTF) $\sigma_{\mathrm{TTF}}$ are known, the distribution may be extrapolated to operational conditions when the more important times to $0.1 \%$ or $0.01 \%$ failure $\left(t_{0.1}\right.$ or $\left.t_{0.01}\right)$ may be estimated. ${ }^{7}$ Uncertainty about the validity of the lognormal distribution means that these values are generally regarded as figures of merit, for comparison purposes between different metallization processes, rather than values to be incorporated in computer aided EM design rules. Such rules, as a result, are simple and conservative. ${ }^{8}$

Despite the good fit to experimental data, there is little theoretical evidence in favor of a lognormal assumption and indeed a certain amount against it. However there have been a number of explanations of the observed statistics and these are now considered in turn.

\section{A. Kolmogorov's gradual erosion model}

This model, which displays lognormality as a result of the central limit theorem (CLT), has been suggested as being applicable to EM in a number of different guises. ${ }^{7,9,10}$ The basic argument is that the damage $\nu(t)$ to [resistance $R(t)$ of, void volume $V(t)$ of, or (dis)functionality $\phi(t)$ of] the system increases from an initial as-deposited value $\nu_{0}$ by a factor $1+X_{m}$ during the time interval $(m T, m T+T)$ so that damage $\nu\left(t_{n}\right)\left(=\nu_{n}\right)$ at time $t_{n}(=n T)$ is given by 


$$
\begin{aligned}
\log \left(\nu_{n}\right) & =\log \left(\nu_{0} \prod_{m=0}^{n-1}\left(1+X_{m}\right)\right) \\
& =\log \left(\nu_{0}\right)+\sum_{m=0}^{n-1} \log \left(1+X_{m}\right) .
\end{aligned}
$$

Now [provided that the central limit theorem (CLT) may be applied] whatever the distribution of the $X_{m}$ and hence of the $\log \left(1+X_{m}\right)$, the distribution of $\log \left(\nu_{n}\right)$ is asymptotically normal (i.e., large $n$ ) and hence $\nu_{n}$ is lognormal. For $n$ to be large, the increases through the $X_{m}$ must be small, hence the erosion must be gradual. It is clear, however, that the lognormal distribution is in the damage $\nu_{n}$ to the system, ${ }^{7}$ in the resistance, ${ }^{9}$ or in the void volume ${ }^{10}$ at time $t$ rather than in the failure time.

In each of these cases, the failure time is determined by the time to reach some critical value of damage (functionality, resistance, or void size) and corresponds to the firstpassage time for the problem. It should be added that experimental results show that the critical void size, the void size that leads, say, to a $20 \%$ increase in resistance, depends upon the microstructure under the cathode via and in some cases has also been found to be lognormally distributed. ${ }^{11}$ In addition the distributions obtained using failure thresholds of $10 \%$ and $20 \%$ resistance increase can be significantly different, suggesting that such critical values are perhaps dependent on the interconnect in question. ${ }^{12}$ Such issues aside, first passage time problems generally have no analytical solution; however if $X_{m}$ is sufficiently small, that $\log \left(1+X_{m}\right) \approx X_{m}$ and has mean $0<\bar{X} \ll 1$, then Eq. (1) becomes

$$
\log \left(\nu_{n} / \nu_{0}\right) \approx \frac{\bar{X}}{T} t+\sum_{k=0}^{n-1}\left(X_{k}-\bar{X}\right) \approx \frac{\bar{X}}{T} t+W(t),
$$

where $W(t)$ is a one-dimensional (1D) Brownian motion. In this case $\log \left(\nu_{n} / \nu_{0}\right)$ is described by a Brownian motion with positive drift $(\bar{X} / T)$ and the failure time distribution is known to be the inverse Gaussian. ${ }^{13}$ However, notwithstanding the fact that the lognormality found does not extend to the failure time distribution, the main objection to this explanation of the observed lognormal damage is that it is somewhat contrived. For example, for a given interconnect, no specific process is invoked for the mechanism by which the increases in line resistance are random in time and independent rather than predictable for that interconnect and correlated in time nor is there an explanation as to why the scheme is multiplicative (rather than additive).

\section{B. Arguments based on failure units}

Another argument for lognormality assumes first a failure-unit model on the scale of a single grain and then explains the lognormality simply as the result of the similar distribution of grain (failure-unit) sizes (e.g., Ref. 14). On the other hand, Lloyd and Kitchin ${ }^{15}$ used the failure-unit model on the scale of larger sections of the whole line and the lack of scalability of the lognormal distribution to argue in favor of a multilognormal approach, for which the single lognormal is just a reasonable approximation. However, it has been shown that the failure-unit model does not fit comfortably with EM failure. ${ }^{16}$ The main problems being (i) a sensible definition of what should constitute a failure unit and (ii) the sense in which such units would behave independently - a necessary requirement of the model.

The argument against the lognormal and in favor of a multilognormal distribution ${ }^{15}$ seems flawed if the via plays an important role in line failure as it is based on the notion that a line of length $2 l$ has the same failure sites as its two half-lines of length $l$ connected in series and uses this to obtain a condition on the failure statistics. If $F_{l}(t)$ is the cumulative distribution function for failure times of lines of length $l$, blocked by a via at both ends, this condition may be expressed as

$$
1-F_{2 \ell}(t)=\left[1-F_{\ell}(t)\right]^{2}
$$

This argument runs that, as a lognormal distribution cannot satisfy Eq. (3) for a general line length, the lognormal choice must be wrong. However the $2 l$ line has a single cathode via while, as implied by Eq. (3), the two half-lines each has a cathode via. In other words, and as is supported by most experimental results, the fact that both distributions are roughly lognormal means that we should conclude that Eq. (3) is not applicable in this case, rather than vice versa. Indeed, for a system in which void nucleation at the cathode, and subsequent growth to a critical size, plays the dominant role in failure statistics, it is fairly clear that $F_{2 l}(t) \approx F_{l}(t)$ for large $l$, which contradicts Eq. (3) whatever $F(t)$. Likewise the existence of a Blech length contradicts Eq. (3).

\section{Normally distributed activation energy $E_{A}$ for diffusion}

A third argument for lognormality assumes that, because the stress buildup has a diffusion component (due to the stress gradient) and a drift component (due to the electron current), the failure time is related to either the diffusion time constant which is $O\left(L_{C}^{2} / \kappa\right)$, where $L_{C}$ is a characteristic length (often associated with the appropriate Blech length or the line length) and $\kappa$ is an effective diffusivity, or to the drift time constant $L_{C} / v$, where the EM drift velocity $v$ is also proportional to $\kappa$ via an Einstein relationship. Under this assumption one obtains a lognormal distribution of failure times on the basis of a normal distribution of (effective) diffusion activation energies ${ }^{15}$ and/or inverse thermal energies $(k T)^{-1} \cdot{ }^{17}$ What is not clear in such models is the origin of the dominant cause of the randomness in the activation energy and thus the correlation length over which it operates. Is it caused by a variation in the crystal orientation of the copper grains and the quality of the copper/cap-layer interface so formed, leading to a correlation length on the scale of the microstructure, or due to impurities at that interface, or is it due to process variations between and along lines giving a larger correlation length scale. These models tend to assume that a single effective diffusivity $\kappa$ may be applied to each line (or a large segment of each line); this value then determines the failure time for that line, and it is implied that the variation in the single value $\kappa$ between lines gives rise to the lognormal behavior. However the mechanisms for the ran- 
domness will generally lead to as much (or more) intrainterconnect diffusivity variations as interinterconnect diffusivity variation. An important example of this, in the understanding of EM failure statistics, is Ref. 15, which uses a version of the failure-element model (explanation B) with $\kappa$, assumed to be fixed for each failure element but allowed to vary (essentially lognormally) between such elements. The variation is attributed to randomness in the grain boundary activation energy; however the authors conclude, although not definitively, that the length of their failure elements, and hence their assumed length scale over which $\kappa$ is assumed to be constant, is likely to be closer to the Blech length for the line and hence of the order of several tens to hundreds of grain diameters.

Additionally wafer-level isothermal tests (ISOT tests) ${ }^{18}$ do not show the change in the DTTF $\sigma_{\mathrm{TTF}}$ implied by a normal distribution of inverse line temperatures $(k T)^{-1}$. This argument is also guilty of the inconsistency of allowing physical variables (such as the diffusion activation energy) to vary between lines but not along them. The importance of such variations along the lines has been effectively confirmed for copper by the results of Choi et al. ${ }^{1}$ on the orientations of the grains surrounding in-line voids.

\section{Lognormal grain size}

Finally, it has been argued that, for via failure, the lognormal distribution of failure times is related to the size of the grain adjacent to the via. ${ }^{19}$ The argument runs that if the whole grain under the via must be consumed for failure to occur and if the grain size distribution is lognormal then so must be the distribution of critical void sizes $V_{\mathrm{cr}}$. Further, if it is assumed that the failure time $t_{f} \propto V_{\text {cr }}$, then $t_{f}$ will consequently possess the same distribution as $V_{\text {cr }}$ and will thus also be lognormal. Expanding this argument a little, one writes for the rate of increase in void size $V(t)$,

$$
\frac{d}{d t} V(t)=J_{\text {void }}(t) \delta_{I} w \Omega,
$$

where $J_{\text {void }}(t)$ is the vacancy current into the void at time $t . \delta_{I}$ is the effective depth of the copper/cap-layer interface and $w$ is the linewidth so that $\delta_{I} w$ is the cross section through which this vacancy current flows and $\Omega$ is the atomic volume. Then the critical volume for failure and the failure time are related by

$$
V_{\mathrm{cr}}=\delta_{I} w \Omega \int_{0}^{t_{f}} J_{\text {void }}(t) d t .
$$

For argument $\mathrm{D}$ to hold requires the additional assumptions both that $J_{\text {void }}$ is roughly constant in time and also that it does not vary significantly between lines, neither of which appears obvious.

Each of these explanations A-D has shortcomings. However there is likely to be some truth in all of them. In view both of these shortcomings and of the apparent ubiquity of the lognormal distribution, it is clear that further investigation is warranted. Obviously interconnects are created differently. Different postprocessing thermal stresses will certainly affect the time required for a void to nucleate and may intro- duce variation in the growth times. Microstructures with a strong (111) texture are generally found to have increased lifetimes. ${ }^{20}$ In addition, the quality of the interface between the copper and the cap layer, which dominates the migration path, is likely to vary between and along lines. Consequently, in the present paper we begin by assuming that void growth occurs according to Eq. (4) and that the failure time is governed by Eq. (5). It is likely that the detailed microstructure under the via will determine the size $V_{\text {cr }}$ to which the void must grow before failure occurs; however it will also determine the current $J_{\text {void }}(t)$ into the void through the same variations in diffusivity noted by Choi et al. ${ }^{1}$ So that, in contrast to argument $\mathrm{C}$ above, we shall allow for a variation in the diffusivity values along an interconnect on the scale of the microstructure (rather than on the scale, say, of a failure segment or of process variations) although clearly variations over a longer scale can be regarded simply as a special case. For now we shall ignore the different initial (thermal) stresses in the lines as mostly affecting the nucleation part of the failure process. In this way we seek to shed some light on the origin of the lognormal failure distribution through firstprinciples physical arguments backed up by simulation of a large number of line failures. Naturally, the conclusions made will be somewhat dependent upon the validity of the model assumed and on the values of the physical parameters used.

To be specific, in our analysis we assume that interconnect failure occurs as a result of the following sequence of events. The applied electrical current drives metallization atoms toward the anode which results in a vacancy buildup at the cathode via. A void nucleates when the tensile stress at the cathode reaches some critical level $\sigma_{\mathrm{cr}}$, after some time $t_{\text {nucl }}$ (say), at which point the stress close to the void collapses as the free surface forms. This free surface effectively pins the stress at the void to zero. Additional atomic migration causes the void to grow at a rate determined by the vacancy current across its surface. When the void length (the void volume normalized to the line cross section) reaches a certain critical size $\Delta_{c r}^{\prime}$, and after some further time $t_{\text {growth }}$, the line fails giving a total failure time of $t_{f}=t_{\text {nucl }}+t_{\text {growth }}$. Initially we will keep $\Delta_{\mathrm{cr}}^{\prime}$ constant returning to the effect of its statistics at the end. Thus we assume a simple single mode failure; other types of problem such as in-line voiding may be treated in a similar fashion but will be considered elsewhere.

We consider EM failure of short copper test structures (length $l \leqslant 50 \mu \mathrm{m}$ ) blocked by a barrier material at the cathode end [so that, during nucleation, the vacancy flux $J(x$ $=l, t)=J_{\text {void }}(t)$ is zero] and with a pad (a void or other free surface) at the anode [pinning the tensile stress $\sigma(x=0, t)$ to zero], such as is used in Ref. 21, for example. The model is also likely to be useful as a template for any line of sufficient length that the stress fields at its anode and cathode are decoupled. In the short line case, significant simplification of the failure process appears to be possible. This belief is based on the fact that following the nucleation of a cathode void [which occurs after a time $t_{\text {nucl }}$ that is of the order of the diffusion time constant, ${ }^{22}$ i.e., $\left.O\left(l^{2} / \kappa\right)\right]$ and after a transient time $t_{\text {trans }}$, also $\sim O\left(l^{2} / \kappa\right)$, void growth will occur at a steady 
rate. In the case of small $l$, both $t_{\text {trans }}$ and $t_{\text {nucl }}$ can be made relatively small compared to the estimated time for steady void growth to failure, typically $\sim \Delta_{\text {cr }}^{\prime} / J_{\text {void }}(t \rightarrow \infty) \Omega$. We shall not, in the present work, allow for a variation with position of void location the initial (thermal) stress, the critical stress for nucleation, the effective elastic modulus, the printed linewidth, the effective valence $Z^{*}$, or the critical void size for EM failure. The inclusion of such features is possible and some of these issues are revisited in Secs. VI of this work.

In the interconnect, the overall atomic diffusion coefficient may be written as a sum of contributions, ${ }^{23}$

$$
D_{A}=D_{b}+\frac{(\delta D)_{\mathrm{GB}}}{d_{50}}+\frac{(\delta D)_{\mathrm{Cu}: \mathrm{N}}}{h}+(\delta D)_{\mathrm{Cu}: b}\left(\frac{2}{w}+\frac{1}{h}\right),
$$

where $D_{b}$ is the diffusion coefficient in the bulk material, while $(\delta D)_{\mathrm{GB}},(\delta D)_{\mathrm{Cu}: \mathrm{N}}$, and $(\delta D)_{\mathrm{Cu}: b}$ are the path width $\times$ diffusion coefficient products, respectively, for grain boundary, copper/caplayer interface, and copper/barrier interface. $h$ is the height of the interconnect and $d_{50}$ is the mean metal grain diameter. In copper interface diffusion dominates $D_{A}$.

It is assumed, for the present, that diffusivity variations on the scale of the microstructure are responsible for the variation in failure times rather than to a lack of perfect process control (printed linewidth, effective elastic modulus B, etc.). This latter could potentially be accounted for through additional (effective) diffusivity variations on a scale larger than the grain size. In any event, the microstructure will in general lead to an atomic diffusivity $D_{A}(x)$ which varies with position along the interconnect. In the analysis below it is assumed to be piecewise constant, however this is not a necessary assumption. Importantly at this stage all lines in the sample are assumed to be held at the same constant temperature $T$.

\section{NUMERICAL MODELING OF THE VOID NUCLEATION}

In copper interconnects, it is generally assumed that the void nucleation time is small compared to the time for void growth and this is reflected in our results. In addition the stress which develops in the line during the nucleation phase determines the start condition for the growth phase. Stresses are assumed to evolve according to the 1D model of Korhonen et al. $^{24}$ which may be written, in dimensionless units, as

$$
\chi=\frac{J \Omega L}{D_{A 0}}=-\frac{\kappa_{A}}{\kappa_{A 0}} \exp (\Sigma)\left(\frac{\partial \Sigma}{\partial X}-\alpha L\right) \quad \frac{\partial \Sigma}{\partial \tau}+\frac{\partial \chi}{\partial X}=0 .
$$

$\Sigma(=\sigma \Omega / \mathrm{kT})$ and $\chi$ are the dimensionless stress and vacancy current. Here $\kappa_{A}$ is the (zero stress) effective atomic diffusivity $=b D_{A}$ and is piecewise constant $(b=B \Omega / k T) . L$ is an arbitrary length scale and $\kappa_{A 0}$ is a representative value of $\kappa_{A}$. Dimensionless times and positions are defined as $\tau$ $=\kappa_{A 0} t / L^{2}$ and $X=x / L$, respectively, and $\alpha=Z^{*} q \rho j / k T$, where $j$ is the applied current, $\rho$ is the resistivity, and $q$ is the electronic charge. We define a dimensionless effective diffusivity $K=\kappa_{A} / \kappa_{A 0}$ equal to $K_{k}$ within the $k$ th grain from the anode, a dimensionless critical void volume $\Delta_{\mathrm{cr}}=\Delta_{\mathrm{cr}}^{\prime} / L$, and in all calculations presented here we set $L=2 \mu \mathrm{m}$. We consider the full nonlinear version of Eq. (7) as, for the structures under consideration, linearized versions can lead to inaccurate solutions. ${ }^{25}$ Although such 1D models naturally ignore some aspects of the stress evolution (which may prove to be important), they are simple and have, in the past, often yielded valuable insights for the interpretation of EM data. Equation (7) is solved for $\Sigma(X, \tau)$, with $\Sigma(X, 0)=0$, $\Sigma(0, \tau)=0$, and $\chi(\tau, X=l / L)=0$, using a standard finite difference (FD) scheme, until the stress reaches $\sigma_{\text {cr }}$ (i.e., $\Sigma$ $=\Sigma_{\mathrm{cr}}$ ) at which point a void is assumed to nucleate. After the void nucleates (at $\tau=\tau_{\text {nucl }}$ ), the stress collapses to zero at the nucleation site, $X=l / L$. This collapse is performed here simply by setting the stress just after nucleation $\left[\Sigma_{\text {nucl }}(X)\right.$ $\left.=\Sigma\left(\tau_{\text {nucl }}^{+}, X\right)\right]$ to be equal to the stress just before nucleation $\left[\Sigma\left(\tau_{\text {nucl }}^{-}, X\right)\right]$, except at the nucleation point $X=l / L$ where $\Sigma$ is set to zero. The stress profile obtained at nucleation is then used as starting point for void growth.

The present analysis has been presented for "short lines" as it may then be expected that the nucleation time is determined by the diffusion time and is $\sim O\left(l^{2} / \kappa\right)$ so that $\tau_{\text {nucl }}$ $\sim O\left(l^{2} / L^{2} K\right)$. The length scale for which this is legitimate may be assessed by neglecting the nonlinearity (i.e., by ignoring the stress dependence of the atomic diffusivity) in Eq. (7) and assuming a homogeneous diffusivity $D_{A}$ throughout the line, straightforward Laplace techniques give the transformed stress at the end point $X=l / L$ to be

$$
\tilde{\Sigma}(x=\ell, s)=\frac{\alpha L \sqrt{K}}{s \sqrt{s}} \tanh \left(\sqrt{s} \frac{\ell}{L}\right) .
$$

If the nucleation occurs rapidly $(s \rightarrow \infty$ and tanh $\sim 1)$ then $\tau_{\text {nucl }}$ satisfies $^{26}$

$$
\alpha^{2} L^{2} K \tau_{\text {nucl }}=\frac{\pi \Sigma_{\mathrm{cr}}^{2}}{4}
$$

so $\tau_{\text {nucl }}$ is independent of $l$, varying rather as $\alpha^{-2}$ or $j^{-2}$. On the other hand inverting Eq. (8) gives, for the stress at the cathode,

$$
\begin{gathered}
\Sigma(X=\ell / L, \tau)=\alpha \ell-\frac{2 \alpha \ell}{\pi^{2}} \sum_{n=0}^{\infty}\left(n+\frac{1}{2}\right)^{-2} \\
\quad \times \exp \left[-\pi^{2} \frac{L^{2}}{\ell^{2}}\left(n+\frac{1}{2}\right)^{2} K \tau\right]
\end{gathered}
$$

or, if the nucleation time is sufficiently large that the first exponential dominates,

$$
\Sigma(X=\ell / L, \tau) \approx \alpha \ell-\frac{8 \alpha \ell}{\pi^{2}} \exp \left(-\frac{\pi^{2} L^{2} K}{4 \ell^{2}} \tau\right),
$$

and the nucleation time $\tau_{\text {nucl }}$ satisfies

$$
\alpha^{2} L^{2} K \tau_{\text {nucl }} \approx-\frac{4 \alpha^{2} \ell^{2}}{\pi^{2}} \log \left[\frac{\pi^{2}}{8}\left(1-\frac{\Sigma_{\text {cr }}}{\alpha \ell}\right)\right] .
$$

For the parameters assumed for copper $\left(\alpha=0.006 \mu \mathrm{m}^{-1}\right.$ and $\left.\Sigma_{\mathrm{cr}}=0.125\right)$, the exact failure time obtained from solving Eq. (10) satisfies Eq. (12) to a very good approximation for $\alpha l$ 


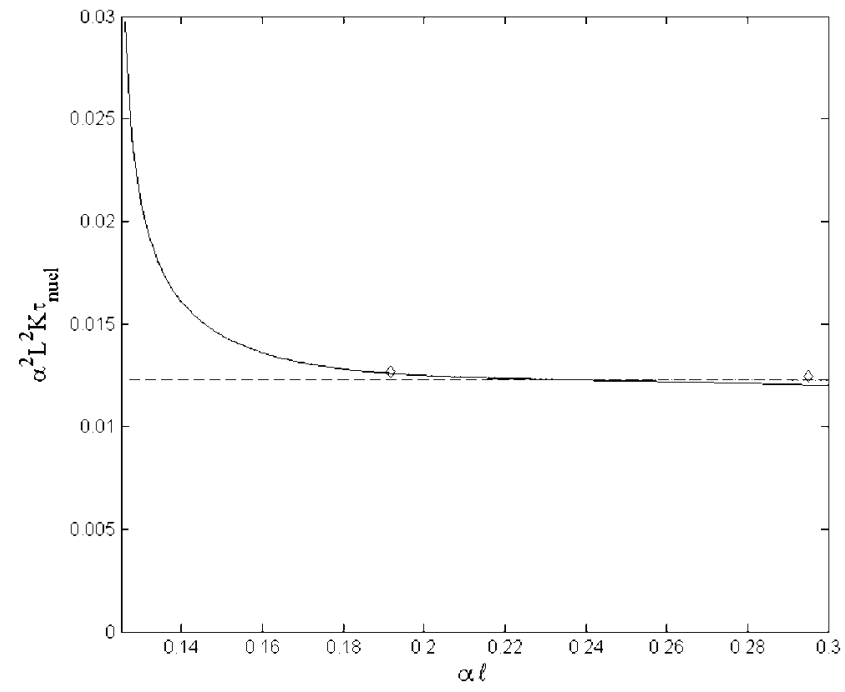

FIG. 1. Plot of $\alpha^{2} L^{2} K \tau_{\text {nucl }}$ as a function of $\alpha l$, obtained from Eq. (12) (solid line) and Eq. (9) (dashed line). Also shown are mean values from calculations of $5050 \mu \mathrm{m}$ lines at $j=20 \mathrm{~mA} \mu \mathrm{m}^{-2}$ and $4050 \mu \mathrm{m}$ lines at $j$ $=13 \mathrm{~mA} \mu \mathrm{m}^{-2}$ obtained by solving the Eq. (7) for nonlinear nonhomogeneous lines using standard FD methods.

$<0.3$ and Eq. (9) for $\alpha l>0.3$ (Fig. 1). The change over limit $\alpha l=0.3$ corresponds to $l \simeq 50 \mu \mathrm{m}$ which is then the upper limit for a short line. Consequently in the present case of short interconnects (less than around $50 \mu \mathrm{m})$, the single exponential expression for $\Sigma(X=l / L, \tau)$ given in Eq. (11) is the appropriate approximation for stress evolution in the homogeneous line.

Also marked in Fig. 1 are the mean values of two sets of nucleation times obtained from the FD solution of Eq. (7),

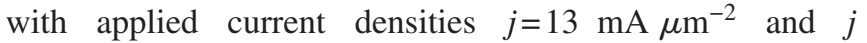
$=20 \mathrm{~mA} \mu \mathrm{m}^{-2}$, and diffusivity values chosen with probabilities $p$ and $(1-p)$ from a slow and a fast lognormal distribution with median values $K_{\text {slow }}$ and $K_{\text {fast }}$ (here arbitrarily we choose $K_{\text {fast }} / K_{\text {slow }}=8$ ) and lognormal deviations both of $\sigma_{D}=0.3$. Clearly the mean values of $\alpha^{2} L^{2} K \tau_{\text {nucl }}$ obtained numerically in this manner lie very close to the theoretical curve. Here $K$ has been replaced by a straightforward average $\left[K_{\text {ave }}=(1-p) K_{\text {fast }}+p K_{\text {slow }}\right]$ of $K_{\text {fast }}$ and $K_{\text {slow }}$ and arbitrarily $p=0.85$.

\section{ANALYSIS OF VOID GROWTH}

Following void nucleation the boundary conditions for the solution of Eqs. (7) become $\Sigma(0, \tau)=\Sigma(l / L, \tau)=0$ with the initial condition of the stress given by $\Sigma(X, 0)=\Sigma_{\text {nucl }}(X)$. As the steady-state condition is one of constant void growth rate, the full nonlinear version of Eq. (7) should be used. ${ }^{25}$ Integrating the second of these equations, between $X$ and $l / L$, gives

$$
\chi(X, \tau)=\chi(\ell / L, \tau)+\frac{\partial}{\partial \tau} \int_{X}^{\ell / L} \Sigma\left(X^{\prime}, \tau\right) d X^{\prime}
$$

Next integrating the first of Eq. (7) over grain $k$ and summing over all grains give

$$
\sum_{k=1}^{N} \frac{1}{K_{k}} \int_{X_{k}}^{X_{k+1}} \chi(X, \tau) \exp (-\alpha L X) d X=1-\exp (-\alpha \ell)
$$

Inserting Eq. (13) into Eq. (14) and simplifying yield

$$
\begin{aligned}
\sum_{k=1}^{N} & \frac{1}{K_{k}}\left(\chi(\ell / L, \tau) \int_{X_{k}}^{X_{k+1}} \exp (-\alpha L X) d X+\frac{\partial}{\partial \tau} \int_{X_{k}}^{X_{k+1}} d X\right. \\
& \left.\times \exp (-\alpha L X) \int_{X}^{\ell / L} \Sigma\left(X^{\prime}, \tau\right) d X^{\prime}\right)=1-\exp (-\alpha \ell) .
\end{aligned}
$$

Finally integrating Eq. (15) over $[0, \tau]$ gives

$$
\begin{aligned}
& Q(\ell / L, \tau) \sum_{k=1}^{N} \frac{\exp \left(-\alpha L X_{i}\right)-\exp \left(-\alpha L X_{i+1}\right)}{\alpha L K_{k}} \\
& \quad+\sum_{k=1}^{N} \frac{1}{K_{k}} \int_{X_{k}}^{X_{k+1}} d X \exp (-\alpha L X) \int_{X}^{\ell / L}\left[\Sigma\left(X^{\prime}, \tau\right)\right. \\
& \left.\quad-\Sigma_{\text {nucl }}\left(X^{\prime}\right)\right] d X^{\prime} \\
& =[1-\exp (-\alpha \ell)] \tau
\end{aligned}
$$

for the (normalized) total amount of material $Q(\ell / L, \tau)$ $=\int_{0}^{\tau} \chi\left(\ell / L, \tau^{\prime}\right) d \tau^{\prime}$ incorporated into the void in the reduced time interval $[0, \tau]$. This then leads directly to the void volume $V(t)$. Here $\Sigma_{\text {nucl }}(X)$ is the stress profile in the interconnect just after void nucleation and represents the initial stress for the growth phase.

Importantly the final term on the left hand side depends only upon $\tau$ through the instantaneous value of $\Sigma(X, \tau)$ and not on its history. Consequently, if the failure time $\tau_{f}$ reaches into the region of steady-state void growth [which it will do for short stripes as the transient approach to steady state (determined by $\delta=\kappa_{A 0} t_{\text {trans }} / L^{2}$ ) can be made sufficiently small by reducing $l]$, we may replace the stress profile by its steady-state value, i.e., for failure times we may use $\Sigma(X, \tau) \sim \Sigma_{\mathrm{SS}}(X)$. Thus, to high accuracy, we have

$$
\begin{aligned}
Q(\ell / L, \tau)= & \frac{[1-\exp (-\alpha \ell)] \alpha L}{\sum_{k=1}^{N} \frac{\exp \left(-\alpha L X_{i}\right)-\exp \left(-\alpha L X_{i+1}\right)}{K_{k}}} \\
& \times\left\{\tau+\frac{\int_{0}^{\ell / L} F\left(X^{\prime}\right)\left[\Sigma_{\text {nucl }}\left(X^{\prime}\right)-\Sigma_{\mathrm{SS}}\left(X^{\prime}\right)\right] d X^{\prime}}{[1-\exp (-\alpha \ell)]}\right\} \\
= & \chi_{\mathrm{SS}}(\tau+\delta),
\end{aligned}
$$

where we have defined the function $F(X)$ $=\int_{0}^{X}\left[\exp \left(-\alpha L X^{\prime}\right) / K\left(X^{\prime}\right)\right] d X^{\prime}$ and changed the order of integration in Eq. (16).

The steady-state current can be obtained from Eq. (17) as 


$$
\begin{aligned}
\chi_{\mathrm{SS}} & =\frac{[1-\exp (-\alpha \ell)]}{\sum_{k=1}^{N} \frac{\exp \left(-\alpha L X_{k}\right)-\exp \left(-\alpha L X_{k+1}\right)}{\alpha L K_{k}}} \\
& =\frac{[1-\exp (-\alpha \ell)]}{F\left(X_{N+1}\right)},
\end{aligned}
$$

while the steady-state stresses are, from Eq. (7),

$$
\Sigma_{\mathrm{SS}}(X)=\alpha L X+\ln \left[1-\chi_{\mathrm{SS}} F(X)\right] .
$$

When $Q(l / L, \tau)$ reaches the critical value of $b \Delta_{\text {cr }}$ failure occurs, thus the time for void growth is given by $b \Delta_{\mathrm{cr}} / \chi_{\mathrm{sS}}-\delta$.
The term $-\delta$ arising as the vacancy current at the point of void nucleation is in general larger than the steady-state value due to the large instantaneous stress gradient at the void surface which causes a rapid initial buildup in void volume. Essentially the reduction arises as $\tau_{\text {nucl }}$, in addition to nucleating a void at $X=l / L$, generates a vacancy distribution which aids void growth as the mass transfer is then not all from $X=0$. As a result, the time for void growth is smaller by $\delta$ than $b \Delta_{\mathrm{cr}} / \chi_{\mathrm{SS}}$.

Before proceeding it is useful to consider the solution obtained by ignoring the nonlinearity, when again for large $\tau$,

$$
Q^{(\operatorname{lin})}(\ell / L, \tau)=\frac{\alpha \ell}{\sum_{k=1}^{N} \frac{X_{k+1}-X_{k}}{K_{k}}}\left\{\tau+\frac{\sum_{k=1}^{N} \frac{1}{K_{k}} \int_{X_{k}}^{X_{k+1}} d X \int_{X}^{\ell / L}\left[\Sigma_{\text {nucl }}\left(X^{\prime}\right)-\Sigma_{\mathrm{SS}}\left(X^{\prime}\right)\right] d X^{\prime}}{\alpha \ell}\right\}=\chi_{\mathrm{SS}}^{(\operatorname{lin})}(\tau+\delta) .
$$

This is also the first term in the small $\alpha$ expansion of Eq. (17). In either case, with the appropriate expression for $\chi_{\mathrm{SS}}$, we may write for the reduced failure time $\tau_{f}$,

$$
\tau_{f}=\tau_{\text {nucl }}+\tau_{\text {growth }}=\frac{b \Delta_{\mathrm{cr}}}{\chi_{\mathrm{SS}}}+\tau_{\text {nucl }}-\delta
$$

As we shall see later [although it is already clear in Fig. 2(c)] the first term in Eq. (21) dominates the failure time in short lines; consequently it is useful to make some comments regarding its statistical properties. In the linear case we may write
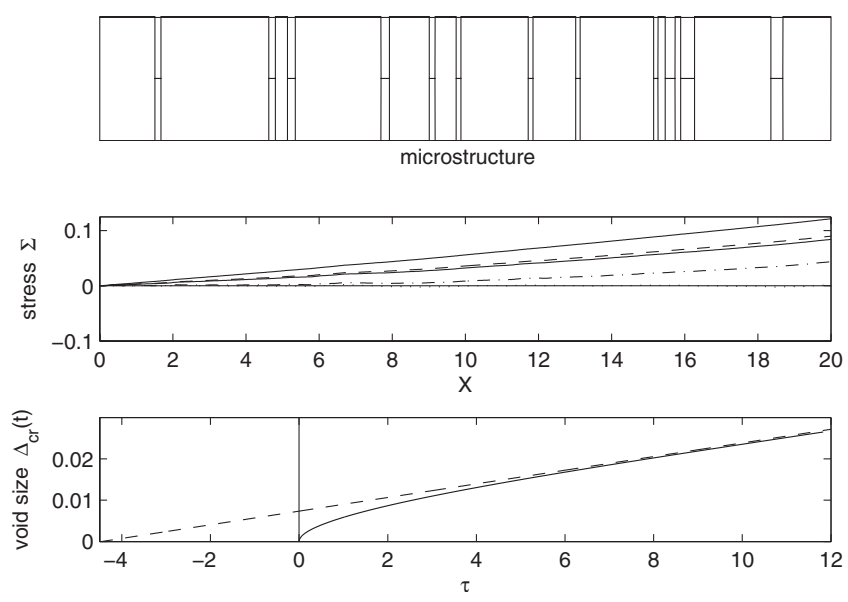

FIG. 2. (a) shows a representative microstructure. The stress evolving in the pad-stud line is shown in (b), while the transient growth of the void volume is shown in (c). Also shown as the dotted line is the steady-state approximation given in Eq. (17). Clearly the approximation gives a very good fit very quickly. Here $l=50 \mu \mathrm{m}$.

$$
\frac{b \Delta_{\mathrm{cr}}}{\chi_{\mathrm{SS}}^{(\operatorname{lin})}}=\frac{b \Delta_{\mathrm{cr}}}{\alpha \ell} \sum_{k=1}^{N} \frac{X_{k+1}-X_{k}}{K_{k}}=\frac{b \Delta_{\mathrm{cr}} D_{A 0}}{\alpha \ell L} \sum_{k=1}^{N} \frac{d_{k}}{D_{A k}} .
$$

For sufficiently large $N$ and for any distribution of the grain diameters $d_{k}$ and atomic diffusivities $D_{A k}$, the sum in Eq. (22) will become normal as a result of the CLT. However there are some distributions for which $N$ must be very large indeed before the sum of $N$ terms approaches normality. One such distribution is the lognormal distribution which for relatively small $N$ [less than many 100 s or even 1000 s (Ref. 27)] shows good lognormal behavior. This is known as the "permanence of the lognormal" and relates to its nonzero skewness. ${ }^{28}$ In most metallization interconnects the grain sizes are lognormal so that for short interconnects Eq. (22) will remain roughly lognormal provided that the $D_{A k}$ values are distributed so. This naturally requires that the activation energy for cap-layer diffusion is distributed roughly normally. With both $d$ and $D_{A}$ lognormal variates, the expression in Eq. (22) is a sum of lognormals (SLN), the properties of which have been examined in some detail. ${ }^{27-30}$ Plotted on lognormal paper the SLN is roughly straight but bends down at small failure times as may be seen in the experiments of $\mathrm{Li}$ et al., ${ }^{10}$ who accounted for the effect rather differently by introducing an additional parameter into the lognormal distribution function. In the nonlinear case of Eq. (18), the fact that $\alpha / \chi_{\mathrm{SS}}$ is a function of $\alpha$ and hence varies with $j$ could have implications for the extrapolation of test conditions to use back conditions, although in practice the dependence of the distribution of $j t_{f}$ on $j$ is small.

\section{FAILURE TIME DISTRIBUTION}

We now seek to demonstrate that for short (pad-stud) copper interconnects the steady-state contribution to void growth does indeed dominate the failure time in both its 
magnitude and its variation. Thus we show that the term $\tau_{\text {nucl }}-\delta$ is small in magnitude and possesses little variation. These remarks partly have their roots in the fact that, in short lines, both $\tau_{\text {nucl }}$ and $\delta$ are related to a single (the largest) diffusion relaxation time for the stripe [similar to that in Eq. (11)] and hence, for a given microstructure, are strongly correlated. As a result we will find that $\tau_{f} \approx b \Delta_{\mathrm{cr}} / \chi_{\mathrm{SS}}$ and consequently, from the above, if the diffusion activation energies are normally distributed and the grain sizes are lognormally distributed, then the failure time will be distributed as a SLN, which is itself roughly lognormal but which shows some bending downward at small failure times when plotted on lognormal probability paper. This implies that if a linear fit on lognormal paper is extrapolated to low failure rates, it will underestimate the reliability rather than overestimate as previously suggested. ${ }^{15}$

The critical parameters for nucleation and failure are assumed to be $\sigma_{\mathrm{cr}} \sim 50-100 \mathrm{MPa}$ (Ref. 31) and $\Delta_{\mathrm{cr}}^{\prime}$ $\sim 150 \mathrm{~nm} ;{ }^{22}$ the acceleration parameters are set at $T$ $=300{ }^{\circ} \mathrm{C}$ with an applied current density of $j$ $=20 \mathrm{~mA} \mu \mathrm{m}^{-2}$ and the material parameters for copper are taken to be $\rho=2.1 \times 10^{-8} \Omega \mathrm{m}, \quad B \sim 28 \mathrm{GPa}, \quad \Omega=1.16$ $\times 10^{-29} \mathrm{~m}^{-3}$, and $q^{*}=0.7 q$ (Ref. 32) for resistivity, effective bulk modulus, atomic volume, and effective charge ( $q$ is the electronic charge), respectively. These assumed values mean that $b=B \Omega / k T \sim 50$ and $\alpha=Z^{*} q \rho j / k T \sim 0.006 \mu \mathrm{m}^{-1}$. With $j=20 \mathrm{~mA} \mu \mathrm{m}^{-2}$, the $j l$ product for a $50 \mu \mathrm{m}$ line is $10^{4} \mathrm{~A} \mathrm{~cm}^{-1}$ which is comfortably greater than that suggested for void nucleation in copper lines. ${ }^{33,34}$ We assume that grain diameters are distributed lognormally, with a median value of $d_{50}=0.5 \mu \mathrm{m}$ and lognormal deviation of $\sigma_{d}=0.36$, values obtained for $0.35 \mu \mathrm{m}$ lines, ${ }^{35}$ although we shall regard these parameters as variables to some extent.

To investigate the statistics of the failure time, we generate a large number of microstructures using a model based around a Bernoulli process ${ }^{36}$ which has been shown to mimic the results of the two-dimensional grain growth simulator MIT/EmSim (Ref. 37) for narrow lines $\left(w \ll d_{50}\right)$. Grains are assigned diameters, drawn from a lognormal distribution and also a diffusivity from either the faster $\left(D_{\text {fast }}\right)$ or slower $\left(D_{\text {slow }}\right)$ distributions with probabilities $q=1-p$ and $p$ ( $p$ $=w / d_{50}=0.85$ is set as the default). All simulations were performed using standard FD methods in MATLAB. ${ }^{38}$ There are roughly 100 grains in each $50 \mu \mathrm{m}$ line, each with its own diffusivity value. Figure 2(a) shows a typical microstructure. Those grains drawn from the fast distribution are indicated by a horizontal line drawn through the middle of the grain.

\section{RESULTS}

Figure 2 shows the results from the numerical simulations for a typical line. The microstructure shown in Fig. 2(a) is determined as in Ref. 36 and the nonlinear version of Eq. (7) is solved on a pad-stud line until the critical stress for void nucleation is achieved. The stress development is shown at a variety of times in Fig. 2(b) while, once the void is formed, the current into the void is shown in Fig. 2(c) as a function of time, reaching a steady-state current after a time characterized by $\delta$. The applied current density is taken to be
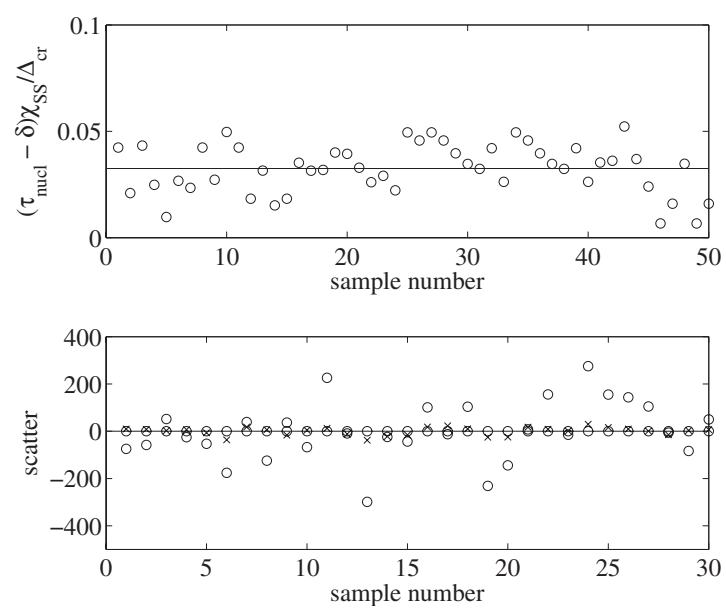

FIG. 3. A comparison of the variation in the failure time due to variations in the nonsteady $\left(\tau_{\text {nucl }}-\delta\right)$ and the steady $\left(\Delta_{\mathrm{cr}} / \chi_{\mathrm{SS}}\right)$ parts of the failure time. (a) shows the ratio of the two for 50 lines of length $50 \mu \mathrm{m}$ at $j$ $=20 \mathrm{~mA} \mu \mathrm{m}^{-2}$. (b) shows the scatter about the mean of $\tau_{\text {nucl }}-\delta$ marked $x$ and of $\Delta_{\mathrm{cr}} / \chi_{\mathrm{sS}}$ marked $o$.

$j=13 \mathrm{~mA} \mu \mathrm{m}^{-2}$. Figure 3(a) shows the ratio ( $\tau_{\text {nucl }}$ $-\delta) /\left(b \Delta_{\mathrm{cr}} / \chi_{\mathrm{SS}}\right)$, while Fig. 3(b) shows the scatter in both $\tau_{\text {nucl }}-\delta$ and $b \Delta_{\mathrm{cr}} / \chi_{\mathrm{SS}}$ about their respective means at an applied current density of $j=20 \mathrm{~mA} \mu \mathrm{m}^{-2}$. It is clear that in both magnitude and in variation the latter term dominates the former. Similar results were obtained for 40 interconnects at $j=13 \mathrm{~mA} \mu \mathrm{m}^{-2}$, the mean of which has been plotted in Fig. 1. As a result it seems fair to make the approximation $\tau_{f}$ $\approx b \Delta_{\mathrm{cr}} / \chi_{\mathrm{SS}}$, i.e., in the linear case,

$$
t_{f} \approx \frac{\Delta_{\mathrm{cr}}^{\prime}}{\alpha \ell} \sum_{k=1}^{N} \frac{d_{k}}{D_{A k}}
$$

which is SLN if the activation energies are normally distributed and thus roughly lognormal..$^{27-30}$ The nonlinear case is only slightly more complicated.

With the approximation in Eq. (23) it is simple to generate statistics for $t_{f}$ using either the linear or the nonlinear versions of Eq. (7). For the remainder of this paper the nonlinear version [i.e., Eq. (18)] is used. In Figs. 4-8 represen-
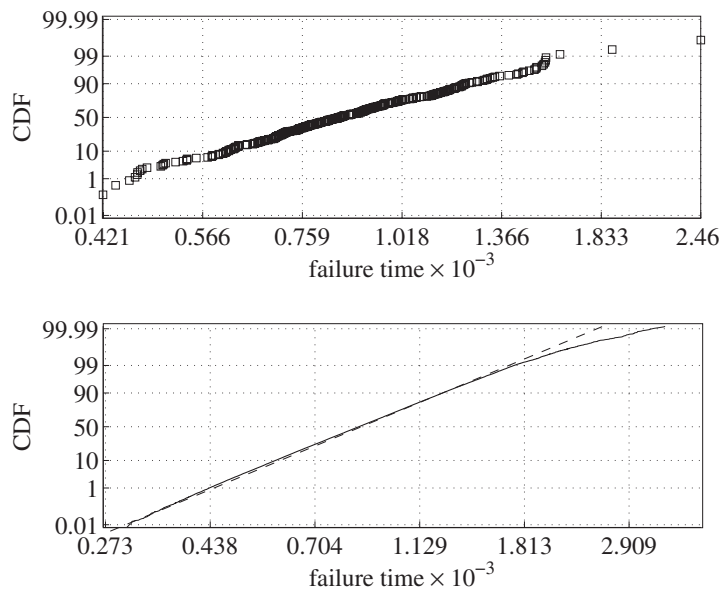

FIG. 4. Lognormal plot of the approximate failure time for (a) 300 lines and (b) $10^{6}$ lines, including the nonlinear terms in Eq. (7). Here $j$ $=20 \mathrm{~mA} \mu \mathrm{m}^{-2}, l=50 \mu \mathrm{m}, \sigma_{D \text { fast }}=\sigma_{D \text { slow }}=0.9$, and $p=0.15$. Times are in arbitrary units as the fast and slow diffusivities are taken as 1 and 0.125 a.u. 


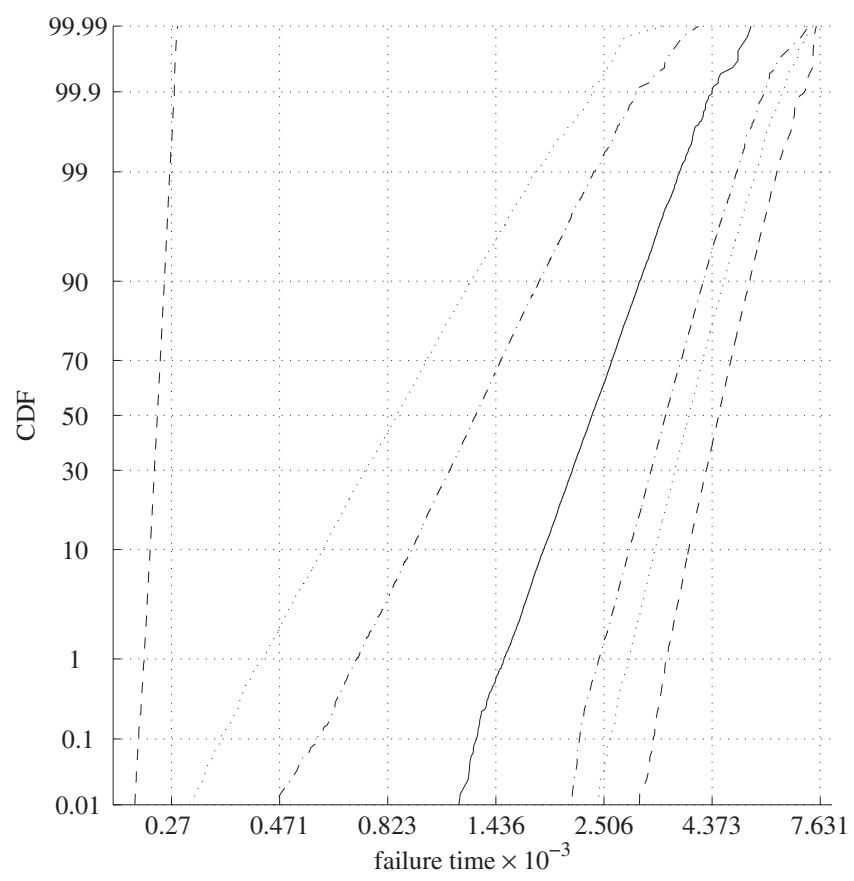

FIG. 5. Variation in the value of $p$. From left to right the curves correspond to $p=0,0.15,0.25,0.50 .75,0.85$, and 1.0 .

tative results are shown assuming the following default conditions: the grain boundaries are distributed so that $\log \left(d_{k} / d_{50}\right) \sim N(0,0.36)$, where $d_{50}$ is $0.5 \mu \mathrm{m}$; the diffusivity is based around fast and slow distributions each with lognormal variance $\sigma_{D}=0.9$ and with relative $D_{50}$ values given by $D_{\text {slow50 }} / D_{\text {fast } 50}=1 / 8$; and the probability of selecting a diffusivity value from the slow distribution is $p=0.85$. First Fig. 4 compares results for 300 lines against that for $1 \times 10^{6}$ lines. The true statistical distribution is naturally the same in each case; however the small sample of 300 lines is

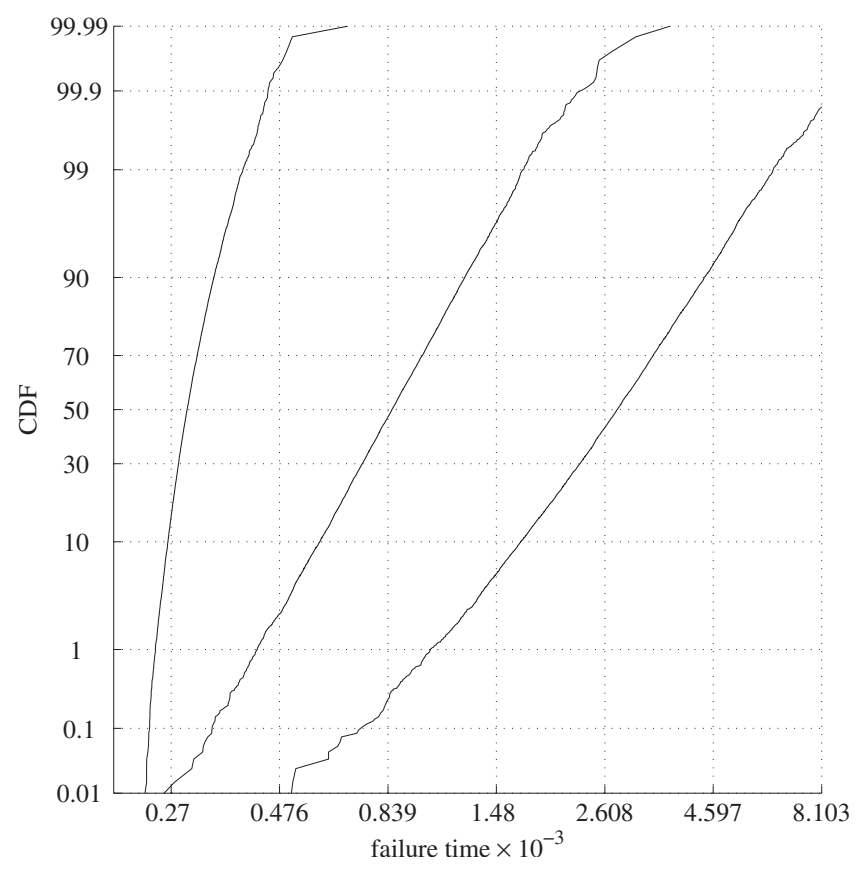

FIG. 6. Variation in the value of $D_{\text {slow50 }} / D_{\text {fast50. }}$. From left to right the curves correspond to $D_{\text {slow50 }} / D_{\text {fast50 }}=1,1 / 8,1 / 32$. Statistics are obtained on $10^{4}$ lines per curve.

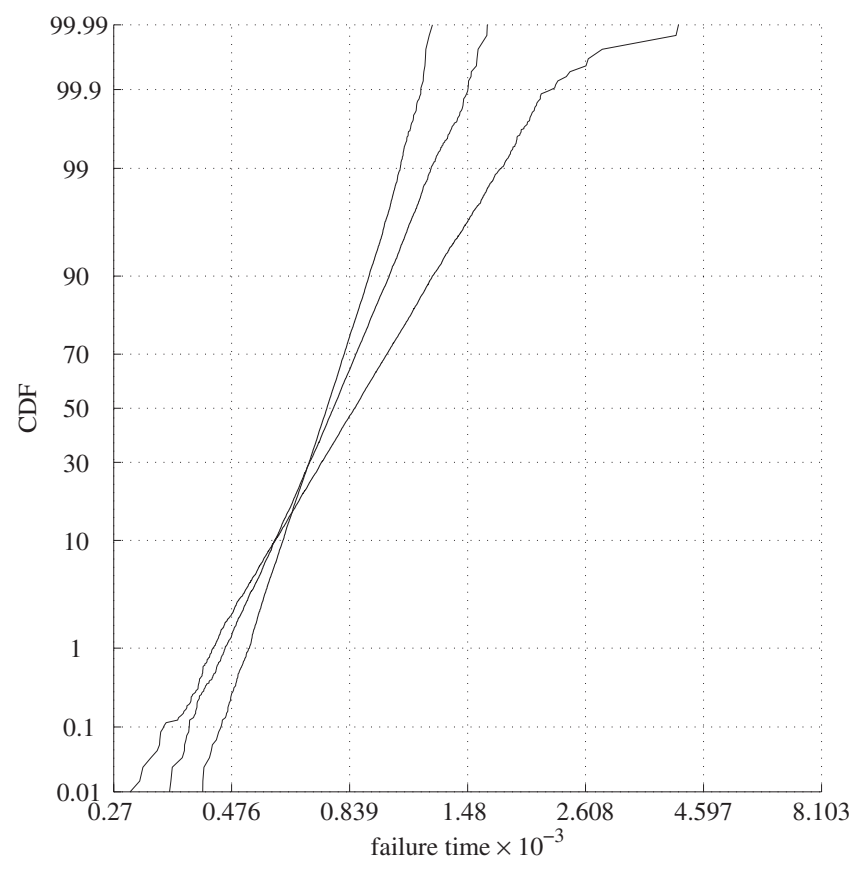

FIG. 7. Variation in the value of $\sigma_{D}$. Both fast and slow populations are assumed to have the same value of lognormal variance. The slope (and hence $\sigma_{\mathrm{TTF}}$ ) increases with $\sigma_{D}$, shown here at values of $0.3,0.6$, and 0.9 . Statistics are obtained on $10^{4}$ lines per curve.

able to hide the slight curvature of the more accurate case of the $1 \times 10^{6}$ lines. The curvature is to be expected in a SLN (Refs. 27-30) distribution and implies that a lognormal fit will underestimate reliability. Figures $5-8$ show the effects of varying the parameters $p$ (the probability of a slow grain), $D_{\text {slow50 }} / D_{\text {fast50 }}$ (the ratio of median values for fast and slow

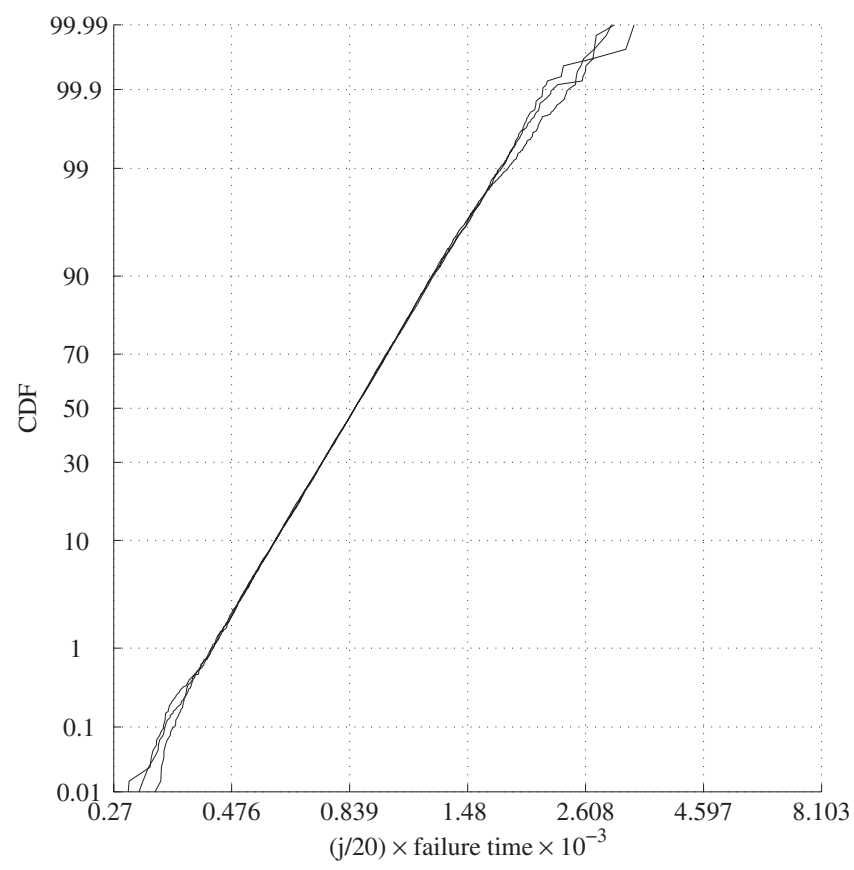

FIG. 8. Variation in the distribution of failure times with applied current density $j$ in $\mathrm{mA} \mu \mathrm{m}^{-2}$. The curves correspond to $j=13,16.5$ and $20 \mathrm{~mA} \mu \mathrm{m}^{-2}$. Smaller currents will not nucleate a void according to the assumptions here and larger currents correspond to the short-time/long-line case. Clearly $j t_{50}$ is roughly constant and there is little dependence of $\sigma_{\text {TTF }}$ on $j$. Statistics are obtained on $10^{4}$ lines per curve. 
diffusions), $\sigma_{D}$ (the lognormal variance of the diffusivity values) which also plays the role of variance of the (assumed normally distributed) diffusion activation energies, and $j$ (the applied current density).

Figure 5 shows the effect of varying $p$ (the composition of the line) for the nonlinear versions of Eq. (7). Clearly as the slow texture improves (increasing $p$ ) the median failure time increases. Figure 6 shows the effect of altering the ratio of median values for fast and slow diffusivities. $D_{\text {slow50 }} / D_{\text {fast50 }}=1$ implies just a single speed with a lognormal variance of $\sigma_{D}=0.9$. Figure 7 shows the effect of varying $\sigma_{D}$ which corresponds to the spread in diffusivity activation energy values. It is clear that an increased $\sigma_{D}$ leads to a larger value of $\sigma_{\mathrm{TTF}}$. Finally, Fig. 8 shows the effect of altering the applied current density $j$. The distribution of $j t_{f}$ is roughly independent of $j$ for the range of values chosen here, although a slight dependence is implied by Eq. (23).

Naturally the results presented here are dependent upon the model used and the parameter values chosen. The parameter space is large and a number of those parameters are not well known. We have used values from the literature where there is general agreement and tried to search the space on those occasions where there is not. Effective diffusivity values in the form $B \Omega \delta_{I} D_{\mathrm{Cu}: \mathrm{N}} / k T$ are generally poorly known so failure times are in arbitrary units. This is not that much of an issue here as it is the shape of the distribution that is important here. The main assumptions regard the use of the stress evolution model of Korhonen et al., ${ }^{24}$ which has been used many times in the past to examine EM behavior and seems a reasonable choice here and also the choice of the distribution of diffusivity values. Here this involved fast and slow distributions with probabilities $(1-p)$ and $p$, respectively. Each distribution is assumed to be lognormal with a lognormal standard deviation $\sigma_{D}$. The values of $p, \sigma_{D}$, and the ratio $r$ of the median values $D_{\text {fast50 }} / D_{\text {slow50 }}$ represent the major unknowns and some search of the space $\left(p, \sigma_{D}, r\right)$ was performed (including $p=0$ and $p=1$ ) (Figs. 4-8). In each case considered the plot gives a reasonable linear fit on lognormal paper.

Many parameters were held fixed throughout the calculations and some restrictions were imposed. Variation in or changes to these parameters may affect our conclusions and we consider some of these now.

\section{CRITICAL VOID SIZE $\Delta_{\text {CR }}^{\prime}$}

We have assumed that the critical void volume is constant. This is not observed experimentally and it is likely that $\Delta_{\mathrm{cr}}^{\prime}$ is a stochastic parameter determined by the microstructure immediately below the cathode via. By contrast the steady-state current is determined by a great many more grains so that it is likely that $\Delta_{\mathrm{cr}}^{\prime}$ and $\chi_{\mathrm{SS}}$ may be considered as being roughly independent stochastic variables. However it is clear that if $\Delta_{\text {cr }}^{\prime}$ were also lognormally distributed (as has been suggested by Hauschildt ${ }^{38}$ ) it will simply add its lognormal variance to $t_{f}$, which will remain roughly lognormal.

\section{A. The long line case $\left(I>2.4 \Sigma_{\mathrm{cr}} / \alpha\right)$}

The present case is that of a short pad-stud line (also covering the case of a line with an in-line via or other free surface within a short distance of the cathode) in which there is a steady state with a nonzero atomic flux and it is this flux that dominates the failure time. As a result the analysis is considerably simplified. In the long line case, it is more difficult to calculate the failure time. However, it is clear from Fig. 1 that the short failure time case of Eq. (9), corresponding to a relatively long line, is roughly equal to the long failure time case of Eq. (12) (short line) when the line length is around the change over length of $l=2.4 \Sigma_{\mathrm{cr}} / \alpha$. Thus a very good approximation to the long line case can be obtained by considering only the last $2.4 \Sigma_{\mathrm{cr}} / \alpha$ of the line and using the short line result [Eq. (21)] as before; thus the sum in Eq. (21) should be restricted to those grains within this distance of the cathode. For short lines in the more realistic stud-stud case, where there is no nonzero steady state, the failure time depends heavily on $\tau_{\text {nucl }}$ and a transient time similar to $\delta$. Such cases are more complicated but can be analyzed in a similar manner, this will be considered in separately.

\section{B. Variation with position of $B, Z^{*}$, and printed linewidth}

The nucleation time will be affected by spatial variations in the elastic modulus B. These will not affect the steadystate current and hence the growth time which dominates $t_{f}$. However the accuracy of the conclusions naturally depends upon the accuracy of the assumed values. If B is smaller than the $28 \mathrm{GPa}$ assumed then the nucleation time will increase as in Eqs. (9) and (12) so the conclusions presented will only strictly apply to shorter lines. However these conclusions may be extended to longer lines as mentioned in A above. A variation in $Z^{*}$ with position simply replaces e.g., Eq. (23) with

$$
t_{f}=\Delta_{\mathrm{cr}}^{\prime} \sum_{k=1}^{N} \frac{d_{k}}{D_{A k}} / \sum_{k=1}^{N} \alpha_{k} d_{k} .
$$

Likewise a variation with position of the printed linewidth will affect the effective diffusivity and the local electron current density (and hence $\alpha$ ), both of which may be incorporated in Eq. (24). If the distributions are narrow they will cause little change, while if they are broad a deviation from lognormal will be seen unless the $\alpha_{k}$ too are lognormally distributed.

Current results are only applicable to the case of a single failure mode in which all failures occur through voids nucleating and growing at the cathode via. The case, again for short pad-stud interconnects, of void nucleation in line followed by void drift to the cathode is considered elsewhere as it requires the development of expressions for the appropriate $\tau_{\text {nucl }}$ and $\delta$. This may be done in terms of the dominant relaxation time for the system using transmission line models of the stress evolution equation of Korhonen et al. ${ }^{24}$. 


\section{CONCLUSIONS}

We have outlined the limitations of several previous arguments explaining the approximate lognormal distribution of EM failure times and provided a new explanation which revolves around a normal distribution of activation energies together with the permanence property of the lognormal distribution-that a SLN is approximately also lognormal. ${ }^{27-30}$ This approach is based on first-principles arguments, backed up by numerical simulations.

Both the time for void nucleation $\left(\tau_{\text {nucl }}\right)$ and the time describing the transient approach to the steady state $(\delta)$ are of the order of the characteristic diffusion relaxation time and consequently show a strong dependence upon the line length $l\left[O\left(l^{2} / \kappa\right)\right]$. Consequently it is possible to set the length $l$ so that $\tau_{\text {nucl }}$ and $\delta$ are much smaller than the time for steady-state growth, which has a much weaker dependence on $l$. For short pad-stud copper test strips the failure time is dominated by the time for steady-state growth provided that $l \leqq 50 \mu \mathrm{m}$. This has been demonstrated using the stress evolution model of Korhonen et al. ${ }^{24}$ together with a simple means of generating line microstructures. ${ }^{36}$ Solving both the full nonlinear $\left[D_{A}(\sigma, x)=D_{A 0}(x) \exp (\sigma \Omega / \mathrm{kT})\right]$ version and the linearized version $\left[D_{A}(\sigma, x)=D_{A 0}(x)\right]$, excellent approximations for the failure time were obtained. In the linearized version, the failure distribution for $t_{f}$ is represented by a SLN provided that the diffusion activation energies are normally distributed. The permanence property of SLNs means that $t_{f}$ is very close to lognormal. A deviation of the SLN from a single lognormal is seen in the important region of early failures where a single (extrapolated) lognormal underestimates the true reliability of the stripe, as opposed to previous work. ${ }^{15}$ The distribution of $j t_{f}$ is independent of $j$, making the extrapolation to operational conditions simple. Solving the nonlinear problem shows additional complication; first the distribution of $j t_{f}$ is no longer entirely independent of $j$, suggesting that the distributions at test and operational conditions may not the be same, although that difference is likely to be small. In addition the failure distribution becomes a fairly complex weighed (W)-SLN. However this too is expected to be roughly lognormal. ${ }^{27-30}$

Including a lognormal distribution of the critical void size $^{38}$ will simply increase the value of the lognormal deviation $\sigma_{\mathrm{TTF}}$ as the distributions of $V_{\mathrm{cr}}$ and $\chi_{\mathrm{SS}}$ can be assumed to be independent of a good first approximation. To summarize, it is quite possible that the observed roughly lognormal distribution of failure times in IC interconnects is due to a broad skewed distribution of diffusivity values varying on the scale of the microstructure.

${ }^{1}$ Z.-S. Choi, R. Mönig, and C. V. Thompson, Appl. Phys. Lett. 90, 241913 (2007).

${ }^{2}$ K. N. Tu, J. Appl. Phys. 94, 5451 (2003).

${ }^{3}$ J. Cho and C. V. Thompson, Appl. Phys. Lett. 54, 2577 (1989).
${ }^{4}$ R. Rosenberg, D. C. Edelstein, C.-K. Hu, and K. Rodbell, Annu. Rev. Mater. Sci. 30, 229 (2000).

${ }^{5}$ M. Ding, G. Wang, B. Chao, P. S. Ho, P. Su, and T. Uehling, J. Appl. Phys. 99, 094906 (2006).

${ }^{6}$ M. H. Lin, Y. L. Lin, K. P. Chang, K. C. Su, and T. Wang, Jpn. J. Appl. Phys., Part 1 45, 700 (2006).

${ }^{7}$ NIST Engineering, Standards handbook. Available on line at http:// www.itl.nist.gov/div898/handbook/apr/section2/apr212.htm.

${ }^{8}$ C. L. Gan, C. V. Thompson, K. L. Pey, W. K. Choi, H. L. Tay, B. Yu, and M. K. Radhakrishnan, Appl. Phys. Lett. 79, 4592 (2001).

${ }^{9}$ C. Ming Tan, N. Raghavan, and A. Roy, J. Appl. Phys. 102, 103703 (2007).

${ }^{10}$ B. Li, C. Christiansen, J. Gill, T. Sullivan, E. Yashchin, and R. Filippi, J. Appl. Phys. 100, 114516 (2006).

${ }^{11}$ M. Hauschildt, M. Gall, S. Thrasher, P. Justison, L. Michaelson, R. Hernandez, H. Kawasaki, and P. S. Ho, "Statistical Analysis of Electromigration Lifetimes and Void Evolution," AIP Conference Proceedings of the Seventh International Workshop on Stress-Induced Phenomena in Metallization, P. S. Ho, S. P. Baker, T. Nakamura, and C. A. Volkert, Vol. 741, Austin, Texas, 14-16 June 2004, pp. 112-123..

${ }^{12}$ B. Li, J. Gill, C. J. Christiansen, T. D. Sullivan, and P. S. McLaughlin, Proceedings of the 43rd International Reliability Physics Symposium (IRPS), 2005 (unpublished), Vol. 43, p. 24.

${ }^{13}$ A. R. Bulsara, in Fluctuations and Order, edited by M. Millonas (Springer-Verlag, New York, 1996), p. 361.

${ }^{14}$ J. H. Park and B. T. Ahn, J. Appl. Phys. 93, 883 (2003).

${ }^{15}$ J. R. Lloyd and J. Kitchin, J. Appl. Phys. 69, 2117 (1991).

${ }^{16}$ V. M. Dwyer, J. Phys. D 37, 2035 (2004).

${ }^{17}$ J. R. Lloyd, J. Appl. Phys. 50, 5062 (1979).

${ }^{18}$ K. Leong, A. Yap, B. H. Lim, E. C. Chua, Y. L. Lim, S. Cheung, and K. Sherry, Proceedings of the Ninth IPFA Symposium, 2002 (unpublished), Vol. 9, p. 129.

${ }^{19}$ e.g., S. Luby and I. Vaura, Proceedings of the Seventh International Vacuum Conference and the Third International Conference on Solid Surfaces, Vienna, 1977 (unpublished), p. 2107.

${ }^{20}$ S. Vaidya and A. K. Sinha, Thin Solid Films 75, 253 (1981).

${ }^{21}$ A. V. Vairagar, S. F. Mhaisalkar, and A. Krishnamoorthy, Thin Solid Films 462-463, 325 (2004).

${ }^{22}$ J. He and Z. Suo, Proceedings of the International Workshop on StressInduced Phenom in Metal, 2004 (unpublished), Vol. 7, p. 1.

${ }^{23}$ E. Zschech, W. Blum, I. Zienert, and P. R. Besser, Z. Metallkd. 92, 803 (2001).

${ }^{24}$ M. A. Korhonen, P. Borgesen, K. N. Tu, and C.-Y. Li, J. Appl. Phys. 73, 3790 (1993).

${ }^{25}$ V. M. Dwyer and W. S. Wan Ismail, J. Appl. Phys. 89, 3064 (2001).

${ }^{26}$ D. T. Walton, H. J. Frost, and C. V. Thompson, Appl. Phys. Lett. 61, 40 (1992).

${ }^{27}$ R. L. Fante, IEEE Trans. Aerosp. Electron. Syst. 37, 739 (2001).

${ }^{28}$ R. Bakarat, J. Opt. Soc. Am. 66, 211 (1976).

${ }^{29}$ N. B. Mehta, J. Wu, A. F. Molisch, and J. Zhang, IEEE Trans. Wirel. Comm. 6, 2690 (2007)

${ }^{30}$ N. C. Beaulieu and F. Rajwani, IEEE Commun. Lett. 8, 709 (2004).

${ }^{31}$ C. S. Hau Riege, S. P. Hau Riege, and A. Marathe, J. Appl. Phys. 96, 5792 (2004).

${ }^{32}$ E. T. Ogawa, K.-D. Lee, V. A. Blaschke, and P. S. Ho, IEEE Trans. Reliab. 51, 403 (2002).

${ }^{33}$ K. D. Lee, E. T. Ogawa, H. Matsuhashi, P. R. Justison, K. S. Ko, and P. S. Ho, Appl. Phys. Lett. 79, 3236 (2001).

${ }^{34}$ S. P. Hau-Riege, J. Appl. Phys. 91, 2014 (2002).

${ }^{35}$ L. Arnaud, G. Taravel, T. Berger, D. Mariolle, Y. Gobil, and I. Touet, Microelectron. Reliab. 40, 77 (2000).

${ }^{36}$ V. M. Dwyer, J. Phys. D 37, 422 (2004).

${ }^{37}$ MATLAB is a registered trademarks of the Mathworks, Inc.

${ }^{38}$ M. Hauschildt, Ph.D. thesis, UT, 2005; Available online at www.lib.utexas.edu/etd/d/2005/hauschildtm39810/hauschildtm39810.pdf. 